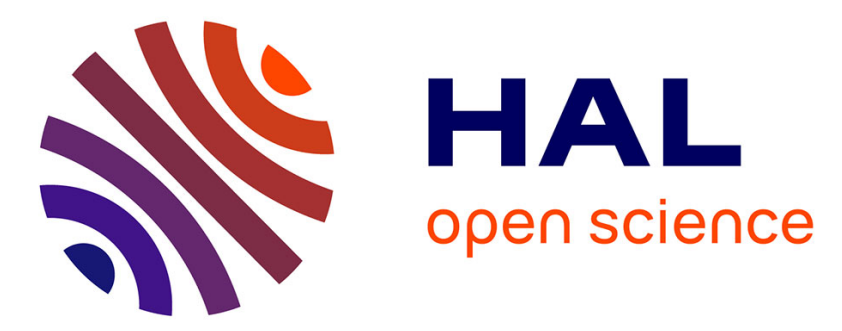

\title{
Instabilités de suspensions anisotropes par passage de courant
}

\author{
M. Adam, P.-G. de Gennes, P. Pieranski
}

\section{To cite this version:}

M. Adam, P.-G. de Gennes, P. Pieranski. Instabilités de suspensions anisotropes par passage de courant. Journal de Physique Lettres, 1978, 39 (4), pp.47-49. 10.1051/jphyslet:0197800390404700 . jpa-00231439

\section{HAL Id: jpa-00231439 https://hal.science/jpa-00231439}

Submitted on 1 Jan 1978

HAL is a multi-disciplinary open access archive for the deposit and dissemination of scientific research documents, whether they are published or not. The documents may come from teaching and research institutions in France or abroad, or from public or private research centers.
L'archive ouverte pluridisciplinaire HAL, est destinée au dépôt et à la diffusion de documents scientifiques de niveau recherche, publiés ou non, émanant des établissements d'enseignement et de recherche français ou étrangers, des laboratoires publics ou privés. 


\title{
LE JOURNAL DE PHYSIQUE-LETTRES
}

\section{Classification}

Physics Abstracts

$62.10-47.20$

\section{INSTABILITÉS DE SUSPENSIONS ANISOTROPES PAR PASSAGE DE COURANT}

\author{
M. ADAM, P. G. DE GENNES et P. PIERANSKI
}

Groupe Hydrodynamique Physique

Ecole Supérieure de Physique et de Chimie, 75231 Paris Cedex 05, France

(Reçu le 17 novembre 1977, révisé le 3 janvier 1978, accepté le 6 janvier 1978)

\begin{abstract}
Résumé. - Dans une suspension de grains anisotropes les coefficients macroscopiques de transport dépendent des gradients de vitesse hydrodynamique. Cet effet peut conduire à des instabilités hydrodynamiques remarquables. L'exemple choisi ici est celui d'une solution d'électrolyte contenant des aiguilles ou des disques en suspension. Dans ces cas favorables, on attend un champ seuil $E^{*}$ pour l'instabilité qui serait seulement de l'ordre de $10 \mathrm{~V} / \mathrm{cm}$.
\end{abstract}

\begin{abstract}
The macroscopic transport coefficients of a suspension made of small rods, or platelets, are sensitive to any hydrodynamic shear rate. This can lead to certain flow instabilities. For instance, an electrolyte containing platelets or rods may, in favourable cases, show an instability at fields $E^{*}$ of order $10 \mathrm{~V} / \mathrm{cm}$.
\end{abstract}

1. Effet d'un cisaillement sur les coefficients de transport. - Nous considérons ici une suspension d'objets anisotropes suffisamment petits pour que le mouvement brownien soit important, et soumis à un écoulement inhomogène caractérisé par le tenseur gradient de vitesse :

$$
s_{\alpha \beta}=\frac{\partial}{\partial x \alpha} v_{\beta}+\frac{\partial}{\partial x \beta} v_{\alpha} .
$$

Le fluide est pris incompressible $\left(s_{\alpha \alpha}=0\right)$ et les composantes $S_{\alpha \beta}$ sont supposées petites $\left(S<D_{\text {rot }}\right)$, où $D_{\text {rot }}$ est le coefficient de diffusion de rotation des grains en suspension [1]. Alors un coefficient de transport comme la conductivité électrique $\sigma_{\alpha \beta}$ doit avoir la forme :

$$
\sigma_{\alpha \beta}=\sigma_{0} \delta_{\alpha \beta}+\sigma_{1} s_{\alpha \beta} .
$$

En effet la partie anisotrope de $\sigma$ est (de même que s) un tenseur symétrique de trace nulle : au premier ordre en $\mathbf{s}$ ces deux tenseurs doivent être proportionnels.

Le coefficient $\sigma_{1}$ est étroitement relié à la biréfringence d'écoulement : le tenseur diélectrique $\varepsilon_{\alpha \beta}$ (à la fréquence optique considérée) a la forme similaire

$$
\varepsilon_{\alpha \beta}=\varepsilon_{0} \delta_{\alpha \beta}+\varepsilon_{1} s_{\alpha \beta} .
$$

Pour un système dilué d'ellipsoïdes de révolution (axe de vecteur unitaire n) on peut relier les perturbations $\varepsilon_{1} s$ et $\sigma_{1} s$ à un tenseur d'alignement

$$
Q_{\alpha \beta}=\frac{1}{2}\left\langle 3 n_{\alpha} n_{\beta}-\delta_{\alpha \beta}\right\rangle
$$

en écrivant

$$
\begin{gathered}
\sigma_{1} s_{\alpha \beta}=\frac{2}{3} Q_{\alpha \beta}\left(\sigma_{\|}-\sigma_{\perp}\right) \\
\varepsilon_{1} s_{\alpha \beta}=\frac{2}{3} Q_{\alpha \beta}\left(\varepsilon_{\|}-\varepsilon_{\perp}\right)
\end{gathered}
$$

où $\sigma_{\|}$et $\sigma_{\perp}\left(\varepsilon_{\|}\right.$et $\left.\varepsilon_{\perp}\right)$ sont les conductivités (constantes diélectriques) principales de la suspension complètement alignée. Le calcul de ces quantités est classique [2]. Par exemple, pour des ellipsoïdes très conducteurs (métalliques) avec des facteurs de démagnétisation $N_{\|}$et $N_{\perp}$ (normalisés par $N_{\|}+2 N_{\perp}=1$ ) on a

$$
\sigma_{\|}-\sigma_{\perp}=\sigma_{s} \Phi\left(N_{\|}^{-1}-N_{\perp}^{-1}\right)
$$

où $\sigma_{\mathrm{s}}$ est la conductance du solvant, $\Phi$ la fraction en volume occupée par les ellipsoïdes. Même pour $\Phi$ assez faible, le rapport $\Phi / N_{\|}$peut être de l'ordre de l'unité [4].

On peut utiliser l'équation (5) de deux façons :

a) En reliant $Q_{\alpha \beta}$ à $s_{\alpha \beta}$ par un calcul direct on trouve

$$
Q_{\alpha \beta}=k s_{\alpha \beta} D_{\text {rot }}^{-1}
$$

où $k$ est une constante dépendant de la géométrie des ellipsoïdes [3] ;

b) il peut être souvent plus pratique d'éliminer $Q_{\alpha \beta}$ et de relier la constante $\sigma_{1}$ à une biréfringence d'écoulement mesurée par ailleurs

$$
\sigma_{1}=\varepsilon_{1} \frac{\sigma_{\|}-\sigma_{\perp}}{\varepsilon_{\|}-\varepsilon_{\perp}}
$$


Le signe de $\sigma_{1}$ est important. A partir des résultats connus sur l'alignement par écoulement [3] on peut prévoir que $\sigma_{1}$ est positif :

a) pour des aiguilles très conductrices, dans un liquide peu conducteur (eau salée),

b) pour des disques plats très isolants, dans un liquide peu conducteur (eau salée).

2. Suspensions sous champ électrique. - Nous allons appliquer l'équation (2) au cas d'une suspension de grains soumis à un champ électrique $\mathbf{E}$. Ils seront alignés par deux effets : d'une part les cisaillements hydrodynamiques (éq. (2)) et d'autre part l'action directe du champ E. Il existe en effet des couples sur le grain même si sa constante $\varepsilon_{\mathrm{i}}$ est égale à celle du liquide $\varepsilon_{\mathrm{e}}$ [6]. Pour le comprendre pensons par exemple au cas du grain isolant : les lignes de courant dans la solution évitent le grain ; ceci implique l'existence de charges à la surface du grain qui déforment les lignes de champ au voisinage de celui-ci. A ces charges est associé un dipôle $\boldsymbol{\mu}$ et une énergie de couplage $U=-\boldsymbol{\mu} . \mathbf{E} / 2$. Si $\boldsymbol{\mu}$ et $\mathbf{E}$ ne sont pas parallèles il en résulte un couple et un mécanisme d'alignement.

Dans ce paragraphe, nous allons calculer le taux d'alignement $Q_{\alpha \beta}$ dû à cet effet, pour l'ajouter ensuite à la contribution hydrodynamique (éq. (7)). Considérons d'abord un ellipsoïde, soumis à un champ $\mathbf{E}$ parallèle à l'un de ses axes principaux, les milieux intérieur et extérieur étant d'abord isolants. Alors le moment $\boldsymbol{\mu}$ vaut [7]

$$
\boldsymbol{\mu}=\frac{\Omega \mathbf{E}}{4 \pi} \frac{\varepsilon_{\mathrm{i}}-\varepsilon_{\mathrm{e}}}{\varepsilon_{\mathrm{e}}+N\left(\varepsilon_{\mathrm{i}}-\varepsilon_{\mathrm{e}}\right)}=\frac{\Omega \alpha \mathbf{E}}{4 \pi} .
$$

a) Pour passer au cas du grain isolant dans un milieu conducteur, il suffit de faire $\varepsilon_{\mathrm{e}} / \varepsilon_{\mathrm{i}} \rightarrow \infty$ dans cette formule [8], d'où

$$
\alpha=-(1-N)^{-1} \text {. }
$$

b) Pour le cas d'un grain métallique dans une solution moins conductrice, il faut prendre la limite $\varepsilon_{\mathrm{i}} / \varepsilon_{\mathrm{e}} \rightarrow \infty$ dans l'équation (9) [8], d'où

$$
\alpha=N^{-1} \text {. }
$$

L'énergie de couplage $U$, pour un grain dont l'axe $\mathbf{n}$ fait un angle $\theta$ avec $\mathbf{E}$, a la forme

$$
\begin{aligned}
& U=\text { Cte }+\Delta\left(3 \cos ^{2} \theta-1\right) / 2 \\
& \Delta=\frac{\Omega E^{2}}{12 \pi}\left(\alpha_{\perp}-\alpha_{\|}\right) .
\end{aligned}
$$

Un calcul statistique simple montre que à une température $T$ telle que $k_{\mathrm{B}} T \gg \Delta$ le taux d'alignement moyen dû au champ vaut

$$
\left\langle\left(3 \cos ^{2} \theta-1\right) / 2\right\rangle=\Delta / 5 k_{\mathrm{B}} T
$$

ou, en revenant à des axes quelconques

$$
\begin{aligned}
Q_{\alpha \beta} & =\lambda\left(3 E_{\alpha} E_{\beta}-E^{2} \delta_{\alpha \beta}\right) / 2 \\
\lambda & =\left(\alpha_{\perp}-\alpha_{\|}\right) \Omega /\left(60 \pi k_{\mathbf{B}} T\right) .
\end{aligned}
$$

L'équation (12) définit complètement l'alignement par un champ faible. En revenant aux équations (10) on voit que $\lambda$ est positif pour des ellipsoïdes allongés, et négatif pour des ellipsoïdes aplatis, et ceci aussi bien que les grains soient isolants ou métalliques.

3. Instabilité électro-hydrodynamique. - Considérons maintenant une suspension soumise à un champ $E_{0}$ parallèle à $O z$ et supposons qu'il n'y a pas électrophorèse [9] : pas de translation d'ensemble des grains. Etudions une fluctuation locale de charge $\rho(\mathbf{r})$ et les fluctuations résultantes de champ $\mathbf{E}_{1}=\mathbf{E}-\mathbf{E}_{0}$, et de vitesse hydrodynamique $v$. Nous nous limiterons ici à un calcul en milieu infini (pas de conditions aux limites imposées par des parois) et à un mode où $\rho$, $\mathbf{v}$ et $\mathbf{E}_{1}$ dépendent seulement de $x . \mathbf{E}_{1}$ est un champ parallèle à $x$ et lié à $\rho$ par l'équation de Poisson

$$
\frac{\partial E_{1}}{\partial x}=4 \pi \rho / \bar{\varepsilon}_{x x}
$$

où $\varepsilon_{x x}$ est la constante diélectrique macroscopique de la suspension mesurée dans la direction $x$. La vitesse $v$ sera, elle, parallèle à $O z$. La conductance électrique a la forme

$$
\sigma_{\alpha \beta}=\bar{\sigma}_{\alpha \beta}+\frac{2}{3}\left(\sigma_{\|}-\sigma_{\perp}\right) Q_{\alpha \beta}^{(1)}
$$

La partie $\bar{\sigma}$ contient l'effet de $E_{0}$ sur $Q_{\alpha \beta}$. Le terme $Q^{(1)}$ décrit le changement d'alignement induit au 1 er ordre par $E_{1}$ et $v$

$$
Q_{x z}^{(1)}=(3 \lambda / 2) E_{0} E_{1}+k D_{\text {rot }}^{-1} \partial v_{z} / \partial x
$$

$\mathrm{Au}$ premier ordre, l'équation de conservation du courant $J$ donne

$-\operatorname{div} J=4 \pi \bar{\sigma}_{x x} \frac{\partial E_{1}}{\partial x}+\frac{2}{3}\left(\sigma_{\|}-\sigma_{\perp}\right) E_{0} \frac{\partial}{\partial x} Q_{z x}=0$

En utilisant (15) et (13), et en définissant le temps de relaxation diélectrique $\tau_{D}=\bar{\varepsilon}_{x x} /\left(4 \pi \bar{\sigma}_{x x}\right)$ on écrit (16) sous la forme

$$
\begin{aligned}
& \rho / \tau_{\mathrm{D}}+(2 / 3)\left(\sigma_{\|}-\sigma_{\perp}\right) \times \\
& \times\left[(3 \lambda / 2) E_{0}^{2} 4 \pi \rho / \bar{\varepsilon}_{x x}+k D_{\text {rot }}^{-1} E_{0} \frac{\partial^{2} v_{z}}{\partial x^{2}}\right]=0 .
\end{aligned}
$$

Il faut y adjoindre une équation d'équilibre des forces

$$
\eta \frac{\partial^{2} v_{z}}{\partial x^{2}}+\rho E_{0}=0
$$

où $\eta$ est la viscosité de la suspension. [Il n'y a pas de 
terme de pression dans (18) car la force $\mathbf{E}_{0} \rho(x)$ est transverse.] En insérant (18) dans (17) on parvient à la condition d'instabilité

$$
\tau_{\mathrm{D}}\left(\sigma_{\|}-\sigma_{\perp}\right)=E_{0}^{2}\left[2 k /\left(3 \eta D_{\text {rot }}\right)-4 \pi \lambda / \varepsilon_{x x}\right] .
$$

Celle-ci aura une solution réelle $E_{0}=E_{\mathrm{s}}$ si $\bar{\varepsilon}_{x x}$ est supérieur à

$$
\varepsilon_{l}=6 \pi \lambda \eta D_{\text {rot }} k^{-1}=\Omega \eta D_{\text {rot }} /\left(2 k k_{\mathrm{B}} T\right)
$$

$D_{\text {rot }}$ est en gros proportionnel à $k_{\mathrm{B}} T / \eta a^{3}$ où $a$ est l'axe long de l'ellipsoïde [1], alors que $\Omega$ est d'ordre $a b^{2}$ ( $b=$ axe court) pour un ellipsoïde allongé, et $a^{2} b$ pour un ellipsoïde aplati. Donc

$$
\varepsilon_{l} \sim \begin{cases}b^{2} / a^{2} & \text { (allongé) } \\ b / a & \text { (aplati) } .\end{cases}
$$

Dans ces deux cas $\varepsilon_{l}$ est plutôt petit. Il est alors facile de réaliser $\bar{\varepsilon}_{x x}>\varepsilon_{l}$, en particulier avec l'eau pour laquelle $\bar{\varepsilon}_{x x} \sim 80$. Nous considérons que dans l'eau le mécanisme d'alignement par le champ (qui correspond au dernier terme de (19)) est négligeable et qu'il existe bien une instabilité.

4. Discussion. - 1) L'origine physique de l'instabilité est assez proche de l'effet Carr-Helfrich dans les nématiques [10] : si une fluctuation de charge positive $\rho>0$ apparaît près d'un point $\mathrm{P}$ (Fig. 1), il en résulte une force $\rho \mathbf{E}_{0}$ vers le haut et un entraî-

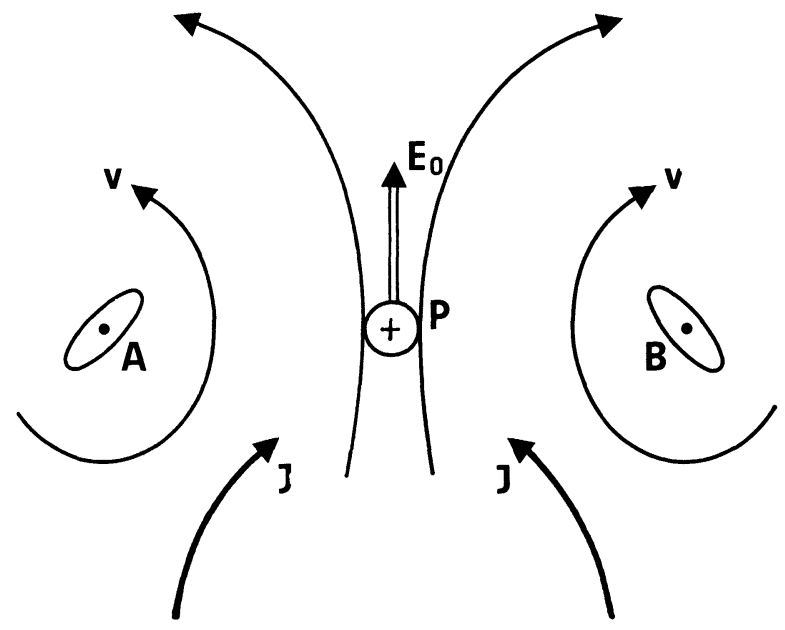

FIG. 1. nement vertical du fluide. Les gradients de vitesse $\partial v_{z} / \partial x$ en $\mathrm{A}$ et $\mathrm{B}$ orientent les grains de la suspension, et ceux-ci défléchissent les courants $\mathbf{J}$ vers la région $P$ : d'où une accumulation de charges + qui augmente la fluctuation initiale.

2) Ordres de grandeur : en utilisant la formule approchée citée plus haut pour $D_{\text {rot }}$ (et en omettant tous les coefficients numériques) on attend d'après l'équation (19) un champ seuil $E_{\mathrm{s}}$ défini par :

$$
E_{\mathrm{s}}^{2} \sim k_{\mathrm{B}} T a^{-3} \bar{\sigma} /\left[\left(\sigma_{\|}-\sigma_{\perp}\right) \bar{\varepsilon}_{x x}\right] .
$$

Pour $\sigma_{\|}-\sigma_{\perp} \sim \sigma_{0}, \bar{\varepsilon}_{x x} \sim 80$, et $a \sim 1 \mu$ on estime ainsi $E_{\mathrm{s}} \sim 10 \mathrm{~V} / \mathrm{cm}$.

3) Si l'on néglige dès le début l'effet d'alignement par le champ les calculs se simplifient, et on peut trouver le champ seuil $E_{\mathrm{s}}(q)$ pour un mode de fluctuation dont le vecteur d'ordre $\mathbf{q}$ est quelconque. Le résultat est

$$
E_{\mathrm{s}}^{2}(q)=E_{\mathrm{s}}^{2} q^{2} /\left(q^{2}-q_{z}^{2}\right) .
$$

Donc c'est bien les modes de $\mathbf{q}$ perpendiculaire à $\mathbf{E}_{0}$ qui sont instables en premier. Pour ceux-ci, le seuil (21) est indépendant de $|\mathbf{q}|$. Cette dégénérescence sera levée à $q$ grand par divers effets :

a) taille finie des aiguilles,

b) existence d'un rayon d'écran $r_{\mathrm{s}}$.

(Notre forme de $J$ ignore un terme de diffusion qui est important pour $q r,>1$ ). Ces corrections seront sans doute nécessaires pour la discussion de l'instabilité entre deux électrodes planes séparées par une distance finie.

4) Les régimes non linéaires au-dessus du seuil ont certains aspects spéciaux si les paillettes sont grandes $\left(D_{\text {rot }}\right.$ petit). En effet, dès que $s \gg D_{\text {rot }}$, l'équation (2) n'est plus valable, et on attend un alignement des grains le long de la vitesse locale $\mathbf{v}$ : ceci tend à réduire le mécanisme d'instabilité.

En conclusion, les suspensions anisotropes nous offrent de nombreuses possibilités d'instabilités nouvelles (le cas du phénomène de Bénard sera traité séparément). Certaines pourraient se rencontrer en génie chimique. D'autres pourraient peut-être servir dans des dispositifs d'affichage lents, utilisant des fluides plus maniables que les nématiques.

\section{Bibliographie}

[1] Perrin, F., Thèse, Paris, Annls de Phys., 1929, J. Physique Radium 7 (1934) 497.

[2] Fricke, H., Phys. Rev. 24 (1924) 575.

[3] Peterlin, A., Stuart, H., Z. Phys. 112 1, 129 ; 113 (1939) 663.

[4] Si l'ellipsoïde est allongé (rapport d'axes $a / b \gg 1$ ) le coefficient $N_{\|} \sim[\ln (2 a / b)-1] b^{2} / a^{2}$, et le rapport $\Phi / N_{\|} \sim \Phi a^{2} / b^{2}$. Par contre les corrections du $2^{\mathrm{e}}$ viriel sur l'équation de bâtons durs sont d'ordre $\Phi a / b$ (voir par ex. réf. [5]). Donc on peut avoir $\Phi / N_{\|} \sim 1$ sans avoir de forts couplages stériques entre grains.

[5] De Gennes, P. G., The Physics of liquid crystals (Oxford) 1974 p. 34 .
[6] Nous sommes redevables à un rapporteur pour cette remarque importante.

[7] Voir par exemple, Bruhat, G., Traité d'électricité (Masson, Paris) 1956

[8] Ces limites $\varepsilon \rightarrow \infty$ peuvent être justifiées en considérant une constante diélectrique complexe $\varepsilon(\omega)=\varepsilon^{\prime}+4 \pi \sigma / i \omega$ et en faisant tendre la fréquence $\omega$ vers 0 .

[9] Ceci peut imposer un ajustement de $\mathrm{pH}$ au point isoélectrique. [10] Helfrich, W., J. Chem. Phys. 51 (1969) 4092. 